\title{
On Estimate of Real Accuracy of EOP Prediction
}

\author{
Zinovy Malkin \\ Institute of Applied Astronomy \\ nab. Kutuzova 10, St.Petersburg 191187, Russia
}

\begin{abstract}
To estimate the real accuracy of EOP predictions, real-time predictions made by the IERS Subbureau for Rapid Service and Prediction (USNO) and at the IAA EOP Service are analyzed. Methods of estimating prediction accuracy are discussed.
\end{abstract}

\section{Introduction}

Forecasts of various time series have been widely used in many fields of science and practice, and many methods have been advanced for the prediction of time series. A common problem of each method is the a priori estimate of its accuracy.

Common practice is to take a truncated series (reference series) ended in the past, investigate its statistical parameters, build its prediction and compare it with existing continuation of series under investigation. Using moving shift of reference series one can collect needed statistics and obtain an estimate of the accuracy of the method depending on the length of prediction. After that, the obtained accuracy is assigned to real predictions.

In most predicted time series, the last observed point (epoch) preceding the first predicted one has its final value and is not the subject of refinement in the future (e.g., number of sunspots for some epoch). It is not the case for EOP. All real EOP predictions are based on operational solution that may differ substantially from final EOP values that comes usually in one-two months.

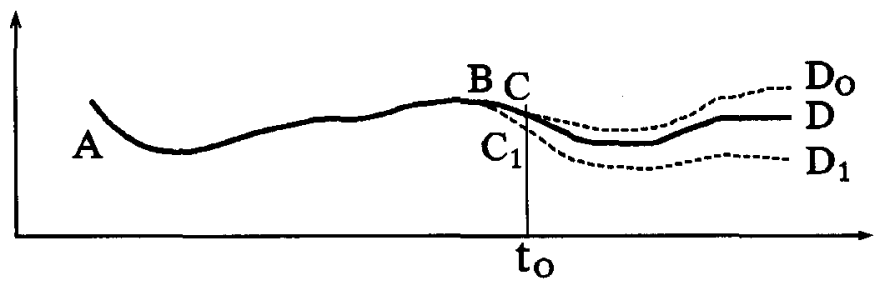

Figure 1. Influence of errors in operational EOP on prediction.

As an illustration of the foregoing, let's consider Fig. 1. In this figure $t_{0}$ is the epoch of the beginning of prediction, $A-B-C_{1}$ is the operational EOP series used to compute predictions, $C_{1}-D_{1}$ is the prediction computed in real time (at date $t_{0}$ ), $A-B-C-D$, the final EOP series, and $C-D_{0}$ is the 
prediction that would be computed from the final series if it were available at date $t_{0}$.

Upon this circumstance, accuracy and other statistical parameters of operational EOP series may differ substantially from ones for final series that are commonly used for a priori estimate of prediction accuracy. It means that estimates of prediction accuracy obtained by "standard" methods may be far from reality and some modification (or at least investigation) is desirable.

\section{A priori estimate of real accuracy of EOP prediction}

Evidently, the most simple way to make an a priori estimate of accuracy of prediction is a simulated perturbation of one or more last points of reference interval to investigate the reaction of a given method of prediction to errors at the last observed epoch(s). This test was performed in (Malkin \& Skurikhina 1996). Two kinds of simulated errors were applied to real observed points:

Test 1: The value of 1 mas was added to (or subtracted from) the $\mathrm{C} 04$ value corresponding to the last observed epoch.

Test 2: The values of $0.5,1.0,1.5$ mas were added to (or subtracted from) the $\mathrm{C} 04$ value corresponding to the three last observed epoch.

This test was used only for an ARIMA method because its influence on the extrapolation of trend-harmonics model (e.g., McCarthy \& Luzum 1991) can be easily foreseen without special calculations. Typical differences between predictions of real and distorted $\mathrm{C} 04$ series are presented in Table 1.

Table 1. Influence of errors in the last values on prediction results.

\begin{tabular}{ccccccccc} 
Test & \multicolumn{8}{c}{ Length of prediction, days } \\
\cline { 2 - 8 } & 1 & 3 & 5 & 10 & 20 & 30 & 60 & 90 \\
\hline 1 & 2.6 & 5.9 & 7.1 & 6.8 & 6.2 & 4.2 & 2.3 & 1.9 \\
2 & 1.2 & 2.2 & 2.2 & 2.1 & 1.9 & 1.7 & 1.4 & 1.2
\end{tabular}

One can see that serious degradation of accuracy may occur when an ARIMA method is used for erroneous observed EOP values. It should be mentioned that this effect practically linearly depends on the value of error. Analogous results were obtained for predictions of UT (Malkin \& Skurikhina 1996).

It is clear that the proposed test can be only useful for the investigation of the sensitivity of a given method of prediction to errors in operational EOP. To estimate a priori errors in real predictions by this method we need (at least) to know real errors in operational EOP series and their statistical parameters.

\section{Assessment of errors in real predictions}

For this investigation we use two series: predictions produced at the IAA EOP Service and ones produced by the IERS Sub-bureau for Rapid Service and Prediction at the USNO. 
First, we can merely compare accuracy of predictions computed at the IAA and the USNO for the period from February 1998 through August 1999. To reduce amount of data only predictions computed on Thursdays were used. Results are presented in Table 2, where column "IAA- $R$ " contains rms errors in predictions computed at the IAA, column "NEOS" contains rms errors in predictions computed at the USNO and published in the IERS Bulletin A. Column "N" contains number of used predictions.

Table 2. RMS errors in predictions (C04 for IAA).

\begin{tabular}{ccccccccc}
\multirow{2}{*}{$\begin{array}{c}\text { Length of } \\
\text { prediction }\end{array}$} & \multicolumn{2}{c}{ IAA-R } & \multicolumn{2}{c}{ NEOS } & \multicolumn{2}{c}{ IAA-A } & \multirow{2}{*}{ N } \\
\cline { 2 - 7 } & $\begin{array}{c}\text { Pole } \\
\text { mas }\end{array}$ & UT & Pole & UT & Pole & UT & \\
& mas & ms & mas & ms & \\
\hline 0 & 0.29 & 0.14 & $\mathbf{0 . 1 9}$ & 0.06 & 0.03 & 0.00 & 76 \\
1 & 0.59 & 0.18 & 0.48 & 0.10 & 0.23 & 0.04 & 76 \\
2 & 0.98 & 0.27 & $\mathbf{0 . 8 5}$ & $\mathbf{0 . 1 7}$ & 0.63 & 0.13 & 76 \\
3 & 1.42 & 0.35 & 1.25 & 0.26 & 1.06 & 0.23 & 76 \\
5 & 2.21 & $\mathbf{0 . 5 9}$ & 1.98 & $\mathbf{0 . 5 0}$ & 1.88 & 0.44 & 76 \\
7 & 2.85 & 0.87 & 2.56 & 0.83 & 2.60 & 0.70 & 75 \\
10 & 3.82 & 1.44 & 3.50 & 1.38 & 3.63 & 1.20 & 75 \\
30 & 9.53 & 5.23 & 9.59 & 5.05 & 9.56 & 5.04 & 73 \\
60 & 15.1 & 10.9 & 15.8 & 11.1 & 15.3 & 10.3 & 69 \\
120 & 21.4 & 25.7 & 25.4 & 28.3 & 21.6 & 25.2 & 60
\end{tabular}

Table 3. RMS errors in predictions (NEOS for IAA).

\begin{tabular}{ccccccccc}
\multirow{2}{*}{$\begin{array}{c}\text { Length of } \\
\text { prediction }\end{array}$} & \multicolumn{2}{c}{ IAA-R } & \multicolumn{2}{c}{ NEOS } & \multicolumn{2}{c}{ IAA-A } & \multirow{2}{*}{ N } \\
\cline { 2 - 7 } & $\begin{array}{c}\text { Pole } \\
\text { mas }\end{array}$ & $\begin{array}{c}\text { UT } \\
\text { ms }\end{array}$ & $\begin{array}{c}\text { Pole } \\
\text { mas }\end{array}$ & $\begin{array}{c}\text { UT } \\
\text { ms }\end{array}$ & $\begin{array}{c}\text { Pole } \\
\text { mas }\end{array}$ & $\begin{array}{c}\text { UT } \\
\text { ms }\end{array}$ & \\
\hline 0 & 0.18 & 0.05 & 0.18 & $\mathbf{0 . 0 5}$ & 0.03 & 0.01 & 48 \\
1 & 0.38 & 0.07 & 0.46 & $\mathbf{0 . 1 0}$ & 0.25 & 0.03 & 48 \\
2 & 0.73 & 0.13 & 0.82 & 0.17 & 0.62 & 0.09 & 48 \\
3 & 1.16 & 0.21 & 1.21 & $\mathbf{0 . 2 7}$ & $\mathbf{1 . 0 4}$ & 0.15 & 48 \\
5 & 1.89 & 0.42 & 1.86 & $\mathbf{0 . 5 3}$ & $\mathbf{1 . 8 9}$ & 0.31 & 48 \\
7 & 2.54 & 0.68 & 2.40 & 0.87 & 2.54 & 0.58 & 47 \\
10 & 3.38 & 1.17 & 3.19 & 1.39 & 3.42 & 1.11 & 47 \\
30 & 8.15 & 5.01 & 7.54 & 4.83 & 8.18 & 5.07 & 45 \\
60 & 11.8 & 11.2 & 11.1 & 11.1 & 11.9 & 11.2 & 41 \\
120 & 16.2 & 24.4 & 19.3 & 24.8 & 16.3 & 24.5 & 32
\end{tabular}

The IAA and the USNO use different methods for short-time prediction (ARIMA at the IAA and trend-harmonics model at the USNO), whereas methods of long-time prediction are very similar (McCarthy \& Luzum 1991, Malkin \& Skurikhina 1996).

In parallel by collecting real-time predictions we computed also a posteriori predictions (in three months after the first day of prediction). Results presented 
in the column "IAA-A" imitate the procedure of a priori estimate of accuracy of prediction.

Table 2 shows that the accuracy of predictions computed at the IAA and the USNO is approximately the same except for the beginning of the interval. Since short-time prediction is the most interesting to users, that is worth investigating more carefully.

Evidently the accuracy of prediction depends on the predicted series. IAA predictions are being made using EOP(IERS)C04 series as reference, whereas USNO uses NEOS series. If there is a substantial difference in accuracy of last epochs of these two operational series, it may cause a difference in the accuracy of the predictions.

To estimate the accuracy of the operational solutions C04 and NEOS we have included in Table 2 the first line with length of prediction equal to zero. This line contains, in fact, the rms error in the last reported epochs of the operational $\mathrm{C} 04$ series in columns related to IAA predictions and the error in the last reported epoch of the NEOS series in the column related to USNO predictions. Comparison of these values shows that the NEOS operational solution is more accurate than the $\mathrm{C} 04$ one.

Comparison of predictions IAA-R and IAA-A shows that accuracy of real predictions differs substantially from a priori estimates for short-time prediction, whereas a priori estimate for long-time prediction is adequate to reality.

Taking into account the difference in accuracy of the operational series used for prediction at the IAA and the USNO we tried to perform another, more rigorous test to compare methods used at these institutions. For this purpose we compute predictions of the NEOS series using the IAA method. In parallel, we computed a posteriori predictions in the same way as above but using the NEOS series as reference, too. Since we began to collect these predictions only in October 1998, statistics for this test are poorer than for the previous one. Table 3 contains results of this test. Notations are the same as in Table 2.

Using results of the last comparison we can conclude that the accuracy of predictions computed at both centers are approximately the same. More detailed investigation requires more predictions involved in statistics. Again, one can see that $a$ posteriori predictions do not provide adequate estimates of the accuracy of short-time predictions.

Another important index of the quality of prediction is the maximum error in predicted EOP values that provide the "guaranteed" error needed in some practical applications. Tables 4 and 5 contains maximum errors in predictions. They are analogous to Tables 2 and 3 . Again, Table 4 contains results for the period from February 1998 through August 1999 and Table 5 from October 1998 through August 1999.

\section{Conclusion}

In this paper we have attempted to estimate the real accuracy of the predictions of EOP using real-time predictions made at the IAA and the USNO. Although collected statistics are too poor to make final conclusions, we can state that:

- Estimate of the accuracy of prediction based on theuse of old data is not adequate to determine the accuracy of real-time prediction, especially for 
Table 4. Maximum errors in predictions (C04 for IAA).

\begin{tabular}{cccccccc}
\multirow{2}{*}{$\begin{array}{c}\text { Length of } \\
\text { prediction }\end{array}$} & \multicolumn{2}{c}{ IAA-R } & \multicolumn{2}{c}{ NEOS } & \multicolumn{2}{c}{ IAA-A } & \multirow{2}{*}{ N } \\
\cline { 2 - 7 } & $\begin{array}{c}\text { Pole } \\
\text { mas }\end{array}$ & $\begin{array}{c}\text { UT } \\
\text { ms }\end{array}$ & Pole & UT & Pole & UT & \\
ms & mas & ms & \\
\hline 0 & 1.09 & 0.40 & 0.63 & 0.18 & 0.21 & 0.01 & 76 \\
1 & 2.82 & 0.51 & 1.34 & 0.29 & $\mathbf{0 . 7 7}$ & 0.11 & 76 \\
2 & 3.96 & 1.02 & 3.00 & 0.52 & 1.65 & 0.34 & 76 \\
3 & 5.39 & 1.09 & 4.08 & 0.87 & 3.42 & 0.50 & 76 \\
5 & 8.31 & 1.83 & 6.34 & 1.78 & 6.54 & 1.07 & 76 \\
7 & 10.1 & 2.70 & 7.65 & 2.72 & 8.37 & 2.09 & 75 \\
10 & 11.7 & 4.33 & $\mathbf{9 . 3 6}$ & 4.40 & 10.6 & 3.65 & 75 \\
30 & 23.2 & 14.4 & 23.0 & 12.1 & 23.6 & 14.1 & 73 \\
60 & 35.1 & 20.2 & 38.4 & 20.4 & $\mathbf{3 4 . 2}$ & 20.3 & 69 \\
120 & 47.5 & 47.0 & 60.1 & 48.8 & 47.4 & 46.5 & 60
\end{tabular}

Table 5. Maximum errors in predictions (NEOS for IAA).

\begin{tabular}{cccccccc}
\multirow{2}{*}{$\begin{array}{c}\text { Length of } \\
\text { prediction }\end{array}$} & \multicolumn{2}{c}{ IAA-R } & \multicolumn{2}{c}{ NEOS } & \multicolumn{2}{c}{ IAA-A } & \multirow{2}{*}{ N } \\
\cline { 2 - 7 } & $\begin{array}{c}\text { Pole } \\
\text { mas }\end{array}$ & ms & Pole & UT & Pole & UT & \\
& ms & mas & ms & \\
\hline 0 & 0.53 & 0.13 & 0.53 & 0.13 & 0.10 & 0.03 & 48 \\
1 & 1.35 & 0.28 & 1.34 & 0.29 & 0.78 & 0.07 & 48 \\
2 & 3.06 & 0.46 & 3.00 & 0.52 & 1.61 & 0.44 & 48 \\
3 & 4.32 & 0.73 & 4.08 & 0.87 & 3.10 & 0.35 & 48 \\
5 & 5.65 & 1.44 & 5.19 & 1.78 & 6.44 & 0.86 & 48 \\
7 & 8.31 & 2.22 & 6.56 & 2.72 & 8.33 & 1.86 & 47 \\
10 & 10.8 & 3.78 & 9.36 & 4.40 & 10.9 & 3.42 & 47 \\
30 & 23.3 & 13.4 & 21.2 & 10.1 & 23.5 & 13.9 & 45 \\
60 & 30.9 & 18.8 & 25.6 & 20.2 & 30.9 & 19.3 & 41 \\
120 & 41.2 & 35.7 & 35.8 & 39.1 & 41.2 & 35.3 & 32
\end{tabular}

short-time prediction. A modification of the commonly used method of $a$ priori estimate of accuracy, e.g., proposed in (Malkin \& Skurikhina 1996) can give more realistic estimates.

- Accuracy of the methods of prediction of EOP used at the IAA and the USNO is approximately the same. More detailed conclusion can be made only after collecting supplemental statistics.

- Estimates of both RMS and maximum errors in prediction are very useful for potential users. It seems reasonable to provide such estimates for IERS and other prediction series. 


\section{References}

Malkin, Z., \& Skurikhina, E. 1996, Comm. IAA, No 93.

McCarthy, D.D. \& Luzum, B.J., 1991, Bull. Géod., 65, 18. 